To Appear in the Astrophysical Journal

Preprint typeset using $\mathrm{LAT}_{\mathrm{E}} \mathrm{X}$ style emulateapj v. 11/10/09

\title{
MULTI-EPOCH ANALYSIS OF PULSE SHAPES FROM THE NEUTRON STAR SAX J1808.4-3658
}

\author{
Sharon M. Morsink ${ }^{1} \&$ Denis A. LeAhY ${ }^{2}$ \\ To Appear in the Astrophysical Journal
}

\begin{abstract}
The pulse shapes detected during multiple outbursts of SAX J1808.4-3658 are analyzed in order to constrain the neutron star's mass and radius. We use a hot-spot model with a small scattered-light component to jointly fit data from two different epochs, under the restriction that the star's mass and radius and the binary's inclination do not change from epoch to epoch. All other parameters describing the spot location, emissivity, and relative fractions of blackbody to Comptonized radiation are allowed to vary with time. The joint fit of data from the 1998 "slow decay" and the 2002 "end of outburst maximum" epochs using the constraint $i<90^{\circ}$ leads to the $3 \sigma$ confidence constraint on the neutron star mass $0.8 M_{\odot}<M<1.7 M_{\odot}$ and equatorial radius $5 \mathrm{~km}<R<13 \mathrm{~km}$. Inclinations as low as $41^{\circ}$ are allowed. The best-fit models with $M>1.0 M_{\odot}$ from joint fits of the 1998 data with data from other epochs of the 2002 and 2005 outbursts also fall within the same $3 \sigma$ confidence region. This $3 \sigma$ confidence region allows a wide variety of hadronic equations of state, in contrast with an earlier analysis (Leahy et al. 2008) of only the 1998 outburst data that only allowed for extremely small stars.

Subject headings: equation of state — pulsars: individual: SAX J1808.4-3658 — gravitation — stars: neutron — stars: rotation — X-rays: binaries
\end{abstract}

\section{INTRODUCTION}

The discovery (Wijnands \& van der Klis 1998) of the first accretion powered millisecond pulsar, SAX J1808.4 3658 (hereafter SAX J1808)), confirms the proposal that neutron stars in low-mass X-ray binaries are the progenitors of millisecond-period pulsars. SAX J1808 is now one of eleven known accreting millisecond X-ray pulsars (see Wiinands (2006) and Poutanen (2006) for reviews). The X-ray pulsations are most likely produced from accretion onto the neutron star's magnetic poles (see, e.g., Fig. 12 of Gierliński et al. (2002)). Spectral models (Gierliński et al. 2002) provide strong evidence that the $\mathrm{X}$-rays correspond to blackbody emission from a spot on the star which is then Compton scattered by electrons above the hot spot. Since the pulsed light is emitted from (or very close to) the neutron star's surface, accreting millisecond X-ray pulsars are excellent targets for lightcurve fitting in order to constrain the neutron star equation of state (EOS). The X-ray light curve depends on the intrinsic properties of the emission spot (size, shape, location, and emissivity), as well as the neutron star's properties (mass, radius, and spin). Constraints on the star's mass and radius lead to constraints on the EOS of supernuclear density material.

The first pulse shape analysis (Poutanen \& Gierliński (2003), hereafter PG03) for SAX J1808 provided interesting constraints on the neutron star's mass and radius. However, this analysis did not take into account two effects that are important for rapidly rotating neutron stars: variable time delays due to light travel time across the star (Cadeau et al. 2005) and the oblate shape of the star (Cadeau et al. 2007). The pulse shape analysis

\footnotetext{
morsink@ualberta.ca, leahy@ucalgary.ca

${ }^{1}$ Department of Physics, University of Alberta, Edmonton, AB, T6G 2G7, Canada

${ }^{2}$ Department of Physics and Astronomy, University of Calgary, Calgary AB, T2N 1N4, Canada
}

including these effects was done by Leahy et al. (2008), resulting in smaller mass and radius than found in PG03. Since the pulse shapes of SAX J1808 were variable during the time period analyzed by PG03 and during subsequent outbursts by SAX J1808 (see the comprehensive analysis by Hartman et al. (2008)), it is important include the time variability in the analysis. In effect, the results of an analysis of a time-averaged pulse profile and an analysis of individually different pulse profiles are expected to be different. Here we carry out an analysis of a set of different pulse profiles from different time periods from SAX J1808. In addition, fitting multiple pulse profiles with the same model should provide a much more stringent test of the validity of the model than fitting of a single pulse profile.

The outline of our paper is as follows. In section 2 , we introduce the different pulse shapes observed from SAX J1808, which come from different phases of the 1998, 2002 and 2005 outbursts. In section 3, the hot spot model is described and we note that an additional scattered-light component is required in order to fit all of the pulse shapes. In section 4 , the results are given for joint fits of the pulse shape data, including resulting mass, radius and inclination values. We conclude with a general discussion of the results, comparing with previous work.

\section{DATA}

SAX J1808 was observed by RXTE during the 1998, 2002, 2005 and 2008 outbursts. In this paper we concentrate on the publicly available data from 1998, 2002 and 2005. Since the pulse shapes detected during the 2008 outburst are very similar to the pulse shapes detected in the earlier outbursts (Hartman et al. 2009), the addition of the 2008 data is not expected to add significant information to the analysis. Hartman et al. (2008) showed that the pulse shape is variable during an outburst and found shorter time periods over which the pulse shape is 
stable, as shown in Figure 3 of their paper. The notation introduced by Hartman et al. (2008) for each time period of an outburst is: 1 , burst rise; 2 , beginning of outburst maximum; 3 , end of the maximum; 4, slow decay; 5, steep luminosity drop; and 6, the flaring tail. We make use of the same time intervals and notation presented by Hartman et al. (2008). For instance, 1998B4 refers to the 1998 outburst during slow decay stage, shown in Box 4 of Figure 3 in Hartman et al. (2008). In our analysis, we can only make use of data that has a high signalto-noise ratio so that meaningful fits to the data can be performed. This results in a total of 7 time intervals, which are used in this paper: 1998B4, 2002B3, 2002B4, 2005B1, 2005B2, 2005B3, and 2005B4. In the case of 2005B4 the data from 2005B4A and 2005B4B were combined in order to provide a stronger signal. The flux from the source is very low during stages 5 and 6 so unfortunately useful pulse shapes are not available. In all cases, the pulse shapes are binned to 32 bins per pulse period.

The data for each time interval are binned into two narrow energy bands, a low-energy band from $3-4 \mathrm{keV}$ and a high-energy band from 9 - $20 \mathrm{keV}$. This choice of energy bands is motivated by previous spectral models Gierliński et al. 2002; Poutanen \& Gierliński 2003) for the pulsed emission that includes two components: blackbody emission and a Comptonized powerlaw. The high-energy band is dominated by the powerlaw component, while the blackbody component makes a significant contribution to the low-energy band. This choice of bands avoids the energy region between 5 and $8 \mathrm{keV}$ which includes the iron line contribution from the disk (Cackett et al. 2009; Papitto et al. 2009).

In this paper we perform joint fits between different time periods. In order to provide the most useful constraints on the model, we need to identify the time intervals in which the pulse shapes are least like each other. To this end, we compute reduced $\chi^{2}$ values comparing each of the 21 possible pairs of time intervals using both energy bands described above with relative normalizations a free parameter. The lowest reduced $\chi^{2}$ is 1.17 (62 degrees of freedom) for the pair 2002B3 and 2005B3 showing that the pulse shapes are not statistically different from each other. The next lowest reduced $\chi^{2}$ is 3.96 which has chance probability $<10^{-16}$. Thus all other pairs of pulse shapes are significantly different. For this reason, we omit the 2005B3 pulse shape from our analysis since it has larger error bars that the 2002B3 pulse shape. The data for the two energy bands for each of these time periods are shown in Figures 11-6. The lowenergy band data are shown using squares in Figures 1 - 6 while circles denote the high-energy band data. The largest reduced $\chi^{2}$ values result from comparisons of the 1998B4 data with any of the other data, ranging from a low of 22.5 (1998B4 and 2005B1) to a high of 63.0 (1998B4 and 2005B4). The large differences can be easily seen by eye: the pulse modulation is about $10 \%$ for the $1998 \mathrm{~B} 4$ data while it is only about $5 \%$ for all of the other time periods. Thus we find that the 1998 outburst pulse shape is significantly different from all of the pulse shapes resulting from the 2002 and 2005 outbursts.

We now compare the data used in this paper with the data used in previous analyses (Poutanen \& Gierliński 2003; Leahy et al. 2008) of SAX J1808. Poutanen \& Gierliński (2003) used data

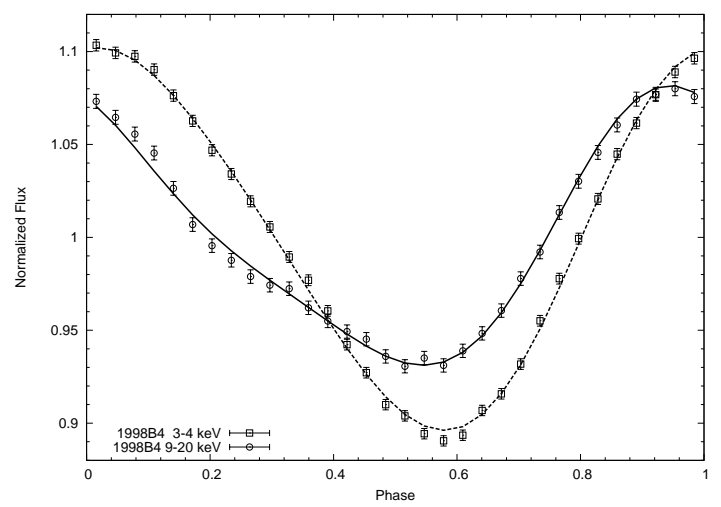

Figure 1. Data from the 1998B4 period of the 1998 outburst (squares 3-4 keV, circles 9-20 keV). The solid and dashed curves correspond to the best-fit model that results from a joint fit of the 1998B4 and 2002B3 data sets. This model (see row 1 of Table 3) has $2 M / R=0.6, M=1.31 M_{\odot}$ and $\chi^{2}=116$ for 110 degrees of freedom.

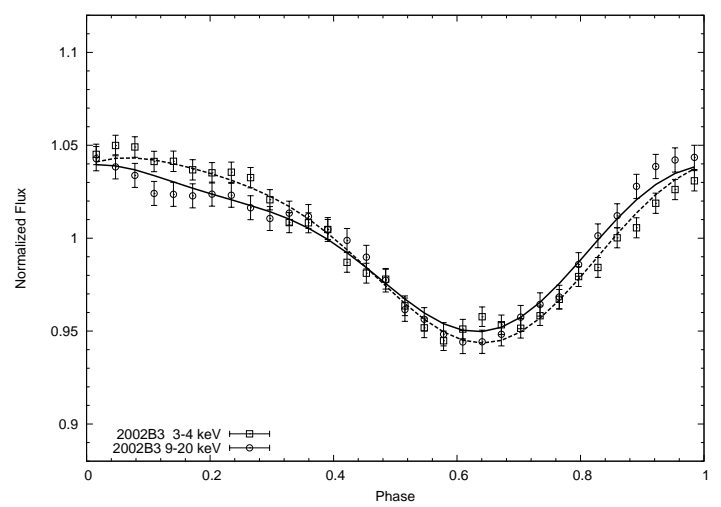

Figure 2. Data from the 2002B3 period of the 2002 outburst (squares 3-4 keV, circles 9-20 keV). The solid and dashed curves correspond to the best-fit model that results from a joint fit of the 1998B4 and 2002B3 data sets. This model (see row 1 of Table 3 has $2 M / R=0.6, M=1.31 M_{\odot}$ and $\chi^{2}=116$ for 110 degrees of freedom.

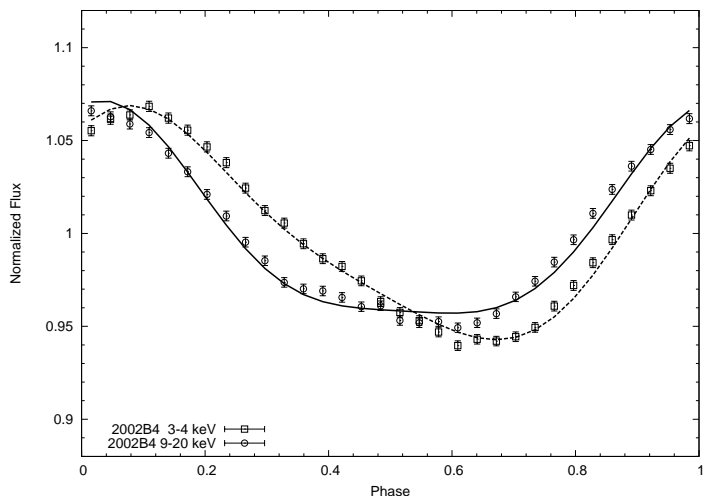

Figure 3. Data from the 2002B4 period of the 2002 outburst (squares 3-4 keV, circles 9-20 keV). The solid and dashed curves correspond to the best-fit model that results from a joint fit of the 1998B4 and 2002B4 data sets. This model (see row 1 of Table 5) has $2 M / R=0.6, M=1.27 M_{\odot}$ and $\chi^{2}=106$ for 110 degrees of freedom. The best-fit light curves for the 1998B4 data are not plotted since the curves are not easily distinguishable from the curves plotted in Figure 1 


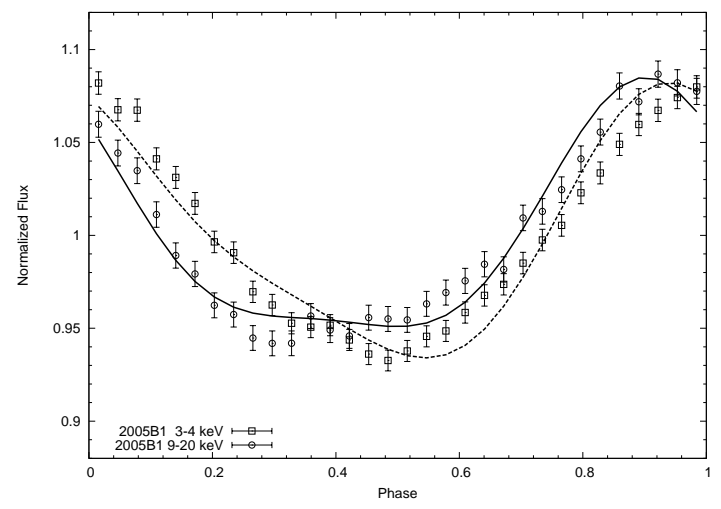

Figure 4. Data from the 2005B1 period of the 2005 outburst (squares 3-4 keV, circles 9-20 keV). The solid and dashed curves correspond to the best-fit model that results from a joint fit of the 1998B4 and 2005B1 data sets. This model (see row 1 of Table 6) has $2 M / R=0.6, M=1.36 M_{\odot}$ and $\chi^{2}=111$ for 110 degrees of freedom.

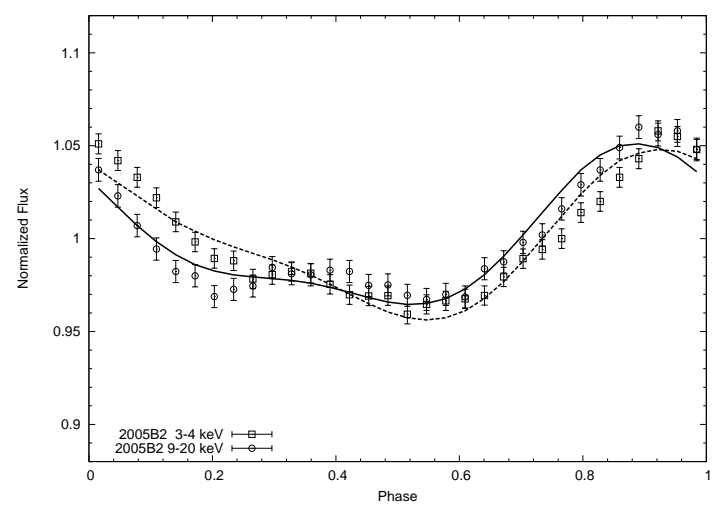

Figure 5. Data from the 2005B2 period of the 2005 outburst (squares 3-4 keV, circles 9-20 keV). The solid and dashed curves correspond to the best-fit model that results from a joint fit of the 1998B4 and 2005B2 data sets. This model (see row 1 of Table 7 ) has $2 M / R=0.6, M=1.20 M_{\odot}$ and $\chi^{2}=109$ for 110 degrees of freedom.

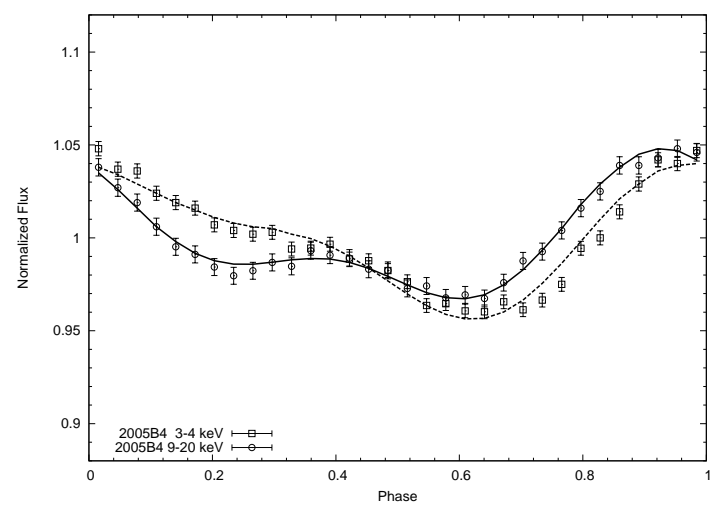

Figure 6. Data from the 2005B4 period of the 2005 outburst (squares 3-4 keV, circles 9-20 keV). The solid and dashed curves correspond to the best-fit model that results from a joint fit of the 1998B4 and 2005B4 data sets. This model (see row 1 of Table 8) has $2 M / R=0.6, M=1.19 M_{\odot}$ and $\chi^{2}=109$ for 110 degrees of freedom. from the 1998 outburst that approximately spans the 1998B4 and 1998B5 time periods, binned into 16 time bins per period, and separated into a 3-4 keV low-energy band and a 12-18 keV high-energy band. In the analysis by Leahy et al. (2008) the same data as Poutanen \& Gierliński (2003) was used, but a bolometric light curve constructed by Papitto et al. (2005) using data from a very short period (22 hours) of the 1998B4 data was also used. The constraints on the mass and radius arising from the bolometric light curve were not very strong compared to the constraints arising from the two-band light curves (see Leahy et al. $(2008))$. In the present analysis, we require time periods over which the pulse shape is stable, thus we use those determined by Hartman et al. (2008). We use pulse shapes with 32 time-bins per period because they show some features that are smoothed out when binned down to 16 time-bins per period. The high-energy band that we use in the present analysis is somewhat wider than the high-energy band used by Leahy et al. (2008). We have confirmed that the change in the high-energy band does not significantly change the best-fit models of the 1998 outburst data. For the pulse shape analysis we added systematic errors equal to the statistical errors, as is common practice.

\section{HOT SPOT MODEL}

The models used in this analysis are similar to those used by Leahy et al. (2008) to model SAX J1808 and by Leahy et al. (2009) to model XTE J1814-338. (These models are similar to that of Poutanen \& Gierliński (2003) for SAX J1808, but include time-delays and oblateness, which are important, e.g. see Morsink et al. (2007)). The model for each pulse shape is composed of three components: (1) Comptonized flux in the highenergy band, (2) Comptonized flux in the low-energy band, and (3) Blackbody flux in the low energy band. For each component, the observed flux, integrated over the energy band, is given by

$$
F_{i}=I_{i} \eta^{3+\Gamma_{i}}\left(1-a_{i} \mu\right)
$$

where the subscript "i" takes on values of 1,2 , or 3 for the 3 components, $I_{i}$ is a normalization, $\eta$ is the Doppler boost factor that depends on the pulse phase, $\Gamma_{i}$ is the effective power-law index for the component, $a_{i}$ is the anisotropy parameter, and $\mu$ is the cosine of the angle between the direction that the photon is emitted and the normal to the surface at the location of the emitter. As in previous models, we take $a_{3}=0$ for the blackbody component, and set $a_{1}=a_{2}=a$ for the Comptonized components. The spectral model fixes the values of the power-law indices $\Gamma_{i}$ as well as the relative normalization of the low-energy band components through the parameter $b=\bar{F}_{3} / \bar{F}_{2}$. In these models, it is assumed that the Comptonized and Blackbody emissions both originate from the same region on the surface of the star.

The flux in each time-bin is computed through the oblate Schwarzschild approximation (Morsink et al. 2007) which accounts for the light-bending caused by gravity, the time-delays caused by the finite travel time for photons across the star and the oblate shape of the rotating star. Since the light-bending depends only on the ratio of the neutron star's mass to radius at the spot location, the star's gravitational radius, $2 G M / R c^{2}$, is kept 
fixed for a series of fits. (For simplicity, when referring to the gravitational radius, we use units with $G=c=1$.) The lowest $\chi^{2}$ for a given $2 M / R$ is found. This allows the most efficient use of "look-up tables" for the lightbending. Once the the ratio of $2 M / R$ has been specified, there are eight free parameters for a fit to one pair of 2-energy band pulse shapes (say 1998B4): R, $\theta, i, I_{1}, I_{2}$, $b, a$, and $\phi$. The angle $\phi$ is an arbitrary phase. The angle between the spot's centre and the spin axis is $\theta$, and the observer's inclination angle is $i$. We assume that the orbital and spin angular momenta are aligned, so that the inclination angle $i$ is the same as the binary's inclination angle. Since the angles $\theta$ and $i$ are both measured from the "north" spin axis, an angle smaller than $90^{\circ}$ corresponds to a spot or observer in the northern hemisphere, while an angle larger than $90^{\circ}$ corresponds to the southern hemisphere. We expect that it is very unlikely that the spot and observer could be in opposite hemispheres, so we restrict our models to ones where $\theta$ and $i$ are both less than $90^{\circ}$. The parameters $a$ and $b$ are not freely varied: the anisotropy is restricted to $0<a<1$ and the parameter $b$ is restricted to be within 1 sigma of the value given by the spectral model.

In this paper we also perform simultaneous fits to the data from two time periods. In these simultaneous fits, the parameters $M, R$, and $i$ are assumed to have the same value in both time periods. All other parameters are allowed to change their values, subject to the restrictions on $\theta, a$ and $b$ mentioned in the previous paragraph. This brings the number of free parameters to 14 .

\subsection{Effect of Spot Size, Shape and Number}

In this paper we restrict the models to only one visible spot. Prior to doing this we carried out fits to the data from all seven time periods with a 2 -spot model. The best fit 2 -spot models in all cases had zero amplitude for the second spot, indicating that no second spot is required by the observed pulse shapes. This is consistent with the model introduced by Ibragimov \& Poutanen (2009) where the accretion disk hides the antipodal spot on the other side of the star during the early stages of the outburst (stages 3 and 4 in the terminology of Hartman et al. (2008); stages $\mathrm{P}$ and SD in the terminology of Ibragimov \& Poutanen (2009)). In the later periods of the burst (stages 5 and 6 ) the disk recedes away from the star in the Ibragimov \& Poutanen (2009) model, exposing the antipodal spot and creating a more complicated pulse shape. The low flux and signal-tonoise in the pulse shapes corresponding to stages 5 and 6 of the 2002 and 2005 outbursts mean that it is not feasible to use these pulse shapes in the present analysis.

In an earlier paper (Leahy et al. 2009), the light curve of a different neutron star, XTE 1814, was analyzed. In that case a one-spot model led to a residual (or bump) in the pulse shape that was seen in all wavelengths. In that case, the residual provided the motivation for a two-spot model for the data which did indeed fit the data significantly better. In the case of SAX J1808, the one-spot models (shown in Section 4) do not lead to a significant excess feature at all wavelengths that would motivate a two-spot model.

Poutanen \& Gierliński (2003) have shown using analytical approximations that the theoretical pulse shape resulting from a hot spot is independent of the spot size, as long as the spot radius is small compared to the size of the neutron star radius. Previous computations of pulse shapes (e.g. Leahy et al. (2008) Leahy et al. (2009)) have confirmed that the assumed spot size does not strongly affect the resulting pulse shape. For this reason, we use a simplified computational procedure that treats the spot size as infinitesimally small. This speeds up the computations considerably compared to the use of spots that cover a larger area. Since the distance to the star and the overall intensity normalization are kept arbitrary, the area of the spot is not needed in our models.

The best-fit models using one infinitesimal spot have unphysically low masses. For example in row 1 of Table 1 we show the best-fit model for the 2002B3 epoch with $2 M / R$ fixed at 0.5 . (Results for other values of the gravitational radius are similar.) Since there are 2 energy bands with 32 time-bins each and a total of 8 free parameters, there are 56 degrees of freedom. The best fit model has a chi-squared value of 69.7 for 56 degrees of freedom, and a mass of only $0.3 M_{\odot}$ which is unreasonably low for neutron stars, or for quark stars. Increasing the radius of the spot so that it has a radius of $0.1 R$ or $0.2 R$ does not change the mass or $\chi^{2}$ significantly, as can be seen in rows 2 and 3 of Table 1 .

We also tested the hypothesis that a non-circular spot could affect the results. We tested a long, thin uniform intensity spot that had a length of $0.4 R$ azimuthally. The result for this extreme shaped spot is shown in row 4 of Table1 where it can be seen that there is again no significant change from the infinitesimal spot. A similar test of a long, thin spot with only latitudinal extent gives similar results. An alterate type of non-circular spot can be approximated by a two spot model where both spots are artifically forced to have the same intensity, but the relative angular locations are allowed to be free. This would allow for two spots to be close together, giving an effective irregular shape. The result for this two-spot model is shown in row 5 of Table 1. This model marginally improves the $\chi^{2}$, but the best-fit mass is still only $0.354 M_{\odot}$. We conclude that changes in the spot size and shape do not help the models to converge to physical values for neutron stars.

\subsection{Scattered-Light Model}

The hot-spot model described in section 3 was used to fit two different data sets simultaneously. The resulting fits were found to be very poor. For example, data from 1998B4 and 2002B3 were fit using this model. The case for $2 M / R=0.5$ is shown in the first row of Table 2 (labelled "No Scatter"). The resulting poor $\chi^{2}$ of 200 for 113 degrees of freedom is typical for fits done for other values of $2 M / R$ and for other pairs of data.

The poor fits that result from the application of the hot-spot model motivates us to introduce an extra component to the model that includes light that travels from the hot-spot on the star, scatters off of material near the star, and then arrives at the observer. The scattering material could be either an accretion column or an accretion disk. If the light is scattered by the disk, then there will be a bright pattern on the disk that will appear to rotate around the star at the same frequency as the star, as studied by Sazonov \& Sunyaev (2001). In this section we will outline a simple model for scattering off 
of an optically thin cloud of electrons, such as the ionized surface layer of an accretion disk.

In this simple model, we do not include the effects of gravitational light-bending or doppler boosting for the scattered light in order to include only the most important features. The reason why we can neglect these normally important relativistic effects is that the amplitude of the scattered light is a very small fraction of the amplitude of the light that travels directly from the spot to the observer. We have tested that the inclusion of Doppler boosting to the scattered light model does not make any significant difference to the outcome of the fits.

Consider a spot at co-latitude $\theta$, azimuthal angle $\phi$ on the surface of a star of radius $R$. The unit vector pointing from the center of the star to the spot is

$$
\hat{n}=\sin \theta(\cos \phi \hat{x}+\sin \phi \hat{y})+\cos \theta \hat{z} .
$$

Photons emitted by the spot are scattered by material at co-latitude $\vartheta$ and azimuthal angle $\varphi$ at a distance $r$. The unit vector pointing to the location of the scatterer is

$$
\hat{r}=\sin \vartheta(\cos \varphi \hat{x}+\sin \varphi \hat{y})+\cos \vartheta \hat{z} .
$$

When light bending is neglected, the photon travels on a straight line defined by the vector $\vec{\ell}=r \hat{r}-R \hat{n}$ which has magnitude

$$
\ell=r\left(\sin ^{2} \sigma+(\cos \sigma-R / r)^{2}\right)^{1 / 2}
$$

where $\sigma$ is the angle between the vectors $\hat{r}$ and $\hat{n}$ defined by

$$
\cos \sigma=\cos \theta \cos \vartheta+\sin \theta \sin \vartheta \cos (\phi-\varphi) .
$$

The vector pointing towards the observer is $\hat{k}=\sin i \hat{x}+$ $\cos i \hat{z}$, so the photon must be scattered through an angle $\rho$ in order to be detected, where $\rho$ is defined by

$$
\begin{aligned}
\cos \rho= & \hat{\ell} \cdot \hat{k}=\frac{1}{\ell}[-R(\cos \theta \cos i+\sin \theta \sin i \cos \phi) \\
& +r(\cos \vartheta \cos i+\sin \vartheta \sin i \cos \varphi)] .
\end{aligned}
$$

The differential cross-section for Thompson scattering is $\frac{d \sigma}{d \Omega} \propto\left(1+\cos ^{2} \rho\right)$ where $\rho$ is the angle that the photon is scattered through. If the flux of light originating from the hot spot at angle $\phi$ that impacts the scatterer at $\varphi$ is $F(\phi)$, the flux of scattered light reaching the observer is then $F_{s c} \propto F(\phi)\left(1+\cos ^{2} \rho\right)$ where the constant of proportionality depends on the optical depth of the scattering material.

In order to model scattering of light by an optically thin cloud of electrons close to the accretion disk, the co-latitude of the scattering material is set to $\vartheta=\pi / 2$. Photons emitted by the spot at angle $\phi=2 \pi t / P+\phi_{0}$ will impact the disk over a range of azimuthal angles $\varphi$ and radii $r$. The pattern appearing on the disk will be centered around an angle $\varphi$ equal to $\phi$. However, the photons emitted by the spot and then scattered by the disk reach the observer at a later time than the photons that travel directly to the observer without scattering. A simple way to treat the time lag is to define an effective phase lag $\Delta \phi$ so that $\varphi=\phi+\Delta \phi$, where $\Delta \phi$ includes the delays due to the light propagation time from the star to the disk as well as the light-crossing times for the photons to cross the disk. The lowest order expression for the phase lag (ignoring relativistic corrections) is

$$
\Delta \phi=2 \pi\left(\frac{r\left(\cos ^{2} \theta+(\sin \theta-R / r)^{2}\right)^{1 / 2}}{c P}-\frac{r}{c P} \sin i \cos \phi\right)
$$

where $P$ is the neutron star's spin period. The time lags for the scattered light should be much less than the star's spin period. Since $c P=750 \mathrm{~km}$ for SAX J1808.4-3658, physical solutions should have a scattering radius $r<<$ $c P$. In this model, we are approximating the extended pattern on the disk as an effective point source. We tested more complicated models with a smeared out pattern on disk and found that the changes from the point source model were insignificant.

The minimum radius of impact on the disk for a photon emitted at co-latitude $\theta$ is $r_{\min }=R / \sin \theta$. Similarly, parts of the disk at radii $r>r_{\text {shadow }}$ can be observed at all values of $\varphi$, where the shadow radius is $r_{\text {shadow }}=$ $R / \cos i$. As a result, the pattern on the disk is always visible to the observer if $\theta \leq \pi / 2-i$. If the spot is at a high latitude (ie., $\theta \ll 1$ ) as suggested by Lamb et al. (2009), then the illuminated part of the disk will be at $r \gg R$ and the illuminated part of the disk will not be eclipsed by the star.

As the spot moves around the star, the resulting pattern on the disk appears to move around the star at the same rate. In the frame rotating with the star, the pattern will not appear to move, so the flux $F(\phi)$ impacting the disk will be a constant. As a result, the scattered flux observed will be proportional to just the differential cross-section. In addition to the 14 parameters required for the hot spot model introduced in the previous section, the scattered light model requires that we add two more free parameters: $I_{s c}$ and $r$. The scattered light is added to the model by adding the term

$$
F_{s c}=I_{s c}\left(1+\cos ^{2} \rho\right)
$$

to each energy band. Since we are implementing a simple model for scattering from a disk $(\vartheta=\pi / 2)$, the scattered flux is

$$
\begin{aligned}
F_{s c}= & I_{s c}\left(1+\left(\frac{r}{\ell}\right)^{2}[\sin i \cos (\phi+\Delta \phi)\right. \\
& \left.\left.-\frac{R}{r}(\cos \theta \cos i+\sin \theta \sin i \cos \phi)\right]^{2}\right),
\end{aligned}
$$

where $\Delta \phi$ depends on the free parameters $r, i, \theta$ through equations (44) and (7). The normalization for the hot spot model is defined so that the amplitudes $I_{1}$ and $I_{2}$ are of order unity, so a physical scattered light model should have an amplitude $I_{s c}<<1$.

In row 2 of table 2 the results of a joint fit to the 1998B4 and 2002B3 data sets using the hot spot model along with the simple scattering model given by equation (9) are shown (labeled "model 1"). Three new parameters were introduced, the flux amplitudes $I_{s 1}, I_{s 2}$ for the scattered light in 1998B4 and 2002B3 and $r$, the effective radius at which the scattering mainly occurs. The addition of these 3 parameters to the model bring the $\chi^{2}$ value down to 120 , which is an acceptable fit for 110 degrees of freedom. The resulting best-fit stellar model has a mass of $1.44 M_{\odot}$. The best-fit spot latitudes are close to the North pole in both time periods, while the inclination 
angle is large, placing the observer close to the equatorial plane of the star. The best-fit scattering radius of $116 \mathrm{~km}$ satisfies both inequalities $r>R / \sin \theta_{1,2}$ and $r>R / \cos i$ so that this location will be illuminated by the spot and will always be visible to the observer. The relative amplitude of the scattered radiation in $1998 \mathrm{~B} 4$ is $0.2 \%$ of the flux coming directly from the spot. Similarly, the relative amplitude for the 2002B3 data is 0.8\%. Both amplitudes are very small fractions of the direct flux, as would be expected for scattered light. The minimum in $\chi^{2}$ is fairly broad in the parameters corresponding the mass of the star and inclination, with the one-sigma allowed region including a range of masses between $1.2-1.6 M_{\odot}$. Only one model (for $2 M / R=0.5$ ) is shown in table 2, but the results are similar for other values of $2 M / R$.

The best-fit solutions using the scattering model described above all have the property that the location of the scatterer is large compared to the radius of the star, $r \gg R$. This motivates the approximation of equation (9) to lowest order in $R / r$,

$$
F_{s c}=I_{s c}\left(1+\sin ^{2} i \cos ^{2}(\phi+\Delta \phi)\right) .
$$

Furthermore, the inclination angles are fairly close to 90 degrees, so $\sin i \simeq 1$. Using this approximation leads to

$$
F_{s c}=I_{s c}\left(1+\cos ^{2}(\phi+\Delta \phi)\right) .
$$

If the best-fit value of $r / R \gg 1$ and $\sin i \sim 1$, equation (11) should be a good approximation to equation (9).

As an alternative to the scattered light model given by equation (9), we now test the simpler model given by equation (11), with $\Delta \phi$ as a free parameter, instead of $r$. The best-fit stellar model that results when equation (11) is added to the basic hot-spot model and fit to the 1998B4 and 2002B3 data sets is shown in row 3 of table 2 (labelled "Model 2"). The best-fit star (with $2 M / R=0.5)$ using Model 2 has $\chi^{2}=120$ for 110 degrees of freedom, so it is as good a fit as Model 1. The best-fit values of the various parameters are different from the values for Model 1, however, the differences are smaller than the one-sigma differences allowed by either model. For instance the mass using Model 2 is $1.57 M_{\odot}$, which lies in the one-sigma region allowed by Model 1 . We conclude that the two models produce results that are statistically the same. For this reason, we use the simpler model using equation (11) to model the scattered light for the rest of this study.

Since the addition of 3 parameters $\left(I_{s 1,2}\right.$ and $\left.\Delta \phi\right)$ brings about a significant reduction in $\chi^{2}$, and the masses are raised to realistic values, we include this scatteredlight model in subsequent fitting of the pulse shapes. In summary, this component is required in order to obtain acceptable fits and to obtain physically reasonable masses.

A small constant (or "DC") flux component was required in a similar analysis of XTE J1814 (see section 3.2 of Leahy et al. (2009)). In the case of XTE J1814, 2 free parameters (one for each energy band) were included to improve the quality of the fits. The amplitudes of the DC components were less than $2 \%$ of the pulsed component, similar to what is seen in the present analysis of SAX J1808.
A previous pulse-shape analysis (Leahy et al. 2008) of the 1998 outburst led to a very low best-fit mass and radius for the neutron star. Although the 1998B4 data are taken over a slightly different time period than the data used by Leahy et al. (2008), a reanalysis of the 1998B4 data does not change the best fit mass and radius by very much. For instance, Table 5 of Leahy et al. (2008) shows the best fit model has a neutron star mass of $0.96 M_{\odot}$ and $\chi^{2} /$ dof $=36.3 / 24$. When we fit the 1998 B4 data including the scattered-light model, we find a best fit mass of $0.98 M_{\odot}$ and $\chi^{2} /$ dof $=63.3 / 54$, which is not significantly different.

An alternative strategy for fitting the data, which should reduce the effects of any near-degeneracies in model parameters, is to do a joint fit between the 1998B4 data and the other data segments. As noted in section 2 , the 1998B4 data has a much higher modulation than the other data segments. In addition, the error bars for the 1998B4 data are relatively small and the data has fairly low scatter compared to most of the other data segments. In the $\chi^{2}$ comparison between the various data streams described in section 2 we note that the largest differences in pulse shape can be found when comparing the 1998B4 data to any of the other data streams. For these reasons it makes sense to attempt to jointly fit the 1998B4 data with the other streams.

For all joint fits we consider two data streams at a time, 1998B4 and one other data set. Due to the large number of parameters needed to model the data, it is not computationally feasible for us to jointly fit more than 2 data streams at a time. In a joint fit, the mass, radius and inclination angle of the neutron star are assumed to be identical in all years. The angular location of the spot $\theta$, anisotropy parameter $a$, blackbody to Compton ratio $b$, scattered-light parameters, normalizations and phases are all allowed to vary with time. This leads to a total of 18 parameters in the joint fits. Since there are 4 light curves (2 energy bands for each time period) with 32 points each, the total degrees of freedom are 110 .

\subsection{Joint Fits to $1998 B 4$ and 2002B3 Data}

The joint analysis of the 2002B3 data with the 1998B4 data results in best-fit neutron star models with larger masses compared to the best-fit models that only use the 1998B4 data. Table 3 shows the values of the best-fit parameters for a range of neutron star compactness values $(2 M / R)$. Each row of Table 3 is generated by keeping the compactness ratio $2 M / R$ (at the location of the spot) at a fixed value and varying all other parameters until the lowest value of $\chi^{2}$ is found. For each value of $2 M / R$ the following variables are displayed: the mass of the neutron star $M$, the equatorial radius of the neutron star $R$, the angular location of the spot $\theta_{1}$ (as measured from the North pole) in 1998, the angular location of the spot $\theta_{2}$ in 2002 , the inclination angle $i$, the anisotropy parameters for $1998\left(a_{1}\right)$ and $2002\left(a_{2}\right)$. We assume the orbital and spin axes are aligned, so $i$ is also the binary's inclination angle. In addition there are 4 normalizations, 2 phases, 2 blackbody to Compton ratios, and 4 scattered-light parameters which are not displayed in the table, since they are of no interest here. A $\chi^{2}$ penalty was used to keep the blackbody to Compton ratios $b_{1}$ and $b_{2}$ within 1 sigma of the spectral models.

The best-fit models in Table 3 should be compared

\section{RESULTS}




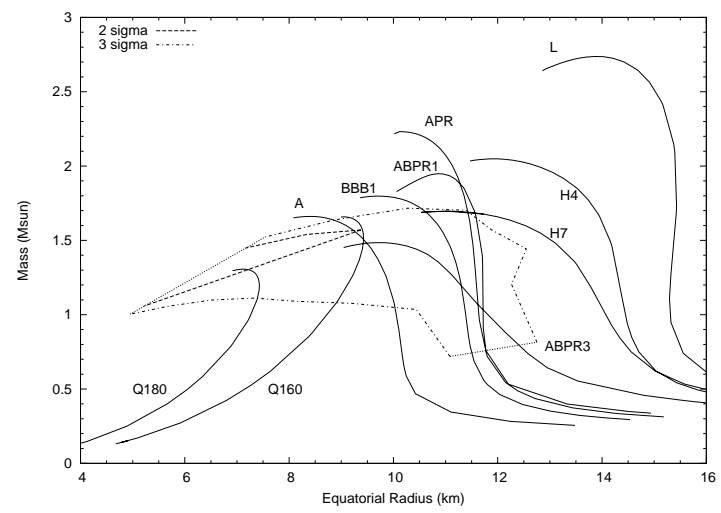

Figure 7. Mass and Radius confidence contours for joint fits to 1998B4 and 2002B3 data using the constraint $i \leq 90^{\circ}$. Massradius curves (solid curves) for stars spinning at a frequency of $401 \mathrm{~Hz}$ are shown for a variety of EOS. Equations of state displayed are: Arnett \& Bowers (1977) models A (Pandharipande 1971) and L (Pandharipande et al. 2006); mixed phase quarkhadron stars ABPR1-3 (Alford et al. 2005); APR (Akmal et al. 1998); BBB1 (Baldo et al. 1997); hyperon stars $\mathrm{H} 4$ and $\mathrm{H} 7$ (Lackev, Navyar, \& Owen 2006); and quark stars Q160 and Q180 (where the number corresponds to value of $B^{1 / 4}$ in $\mathrm{MeV}$ where $B$ is the bag constant Glendenning (2000)). Confidence contours for $2 \sigma$ (dashed curve) and $3 \sigma$ (dot-dashed curve) are shown. The boundaries shown as dotted lines correspond to the largest $(0.6)$ and smallest $(0.2)$ values of $2 M / R$ used in the computations.

with Table 5 of Leahy et al. (2008) where only data for 1998 was used. Consider the best-fit models with $2 M / R=0.4$ in both tables. When only 1998 data was used, the best-fit model had small mass and radius ( $M=0.90 M_{\odot}$ and $\left.R=6.7 \mathrm{~km}\right)$ and both the spot's angular location and the inclination angle were close to 30 degrees. The addition of data from 2002B3 allows for a larger mass and radius $\left(M=1.43 M_{\odot}\right.$ and $\left.R=10.8 \mathrm{~km}\right)$ for the same value of compactness. The larger radius is compensated for by moving the spot closer to the spin axis with $\theta_{1}$ close to 10 degrees in 1998 and 5 degrees in 2002B3.

The best-fit values of the scattered-light amplitudes $A_{1}$ are $2 \times 10^{-3}$ for $1998 \mathrm{~B} 4$ and $8 \times 10^{-3}$ for 2002B3, where the flux is normalized to 1 .

The best-fit model with $2 M / R=0.6$ in Table 3 is shown in Figures 11 and 2 with dashed curves for the low-energy bands and solid curves for the high-energy bands. All four light curves are fit simultaneously, but for clarity, the data and best-fit models are plotted in two separate figures. The relative phases between the high and low energy-bands for each data set are included in the models.

Figure 7 shows 2 and $3 \sigma$ confidence contours on the mass-radius plane for joint fits with 1998B4 and 2002B3. This figure is generated by specifying a mass and then varying all parameters until the lowest value of $\chi^{2}$ for the mass is found. Two constraints were added to the variation of parameters. First, the ratio $2 M / R$ was only sampled in the range $0.2 \leq 2 M / R \leq 0.6$ in order to stay within the bounds of reasonable equations of state. Second, an unconstrained variation of the inclination angle leads to acceptable fits for solutions with $i>90^{\circ}$, which is very unlikely. Hence, we constrained the inclination angle to values with $i \leq 90^{\circ}$. The resulting confidence contours in Figure 7 should be compared with the corresponding confidence contours for the 1998 data shown

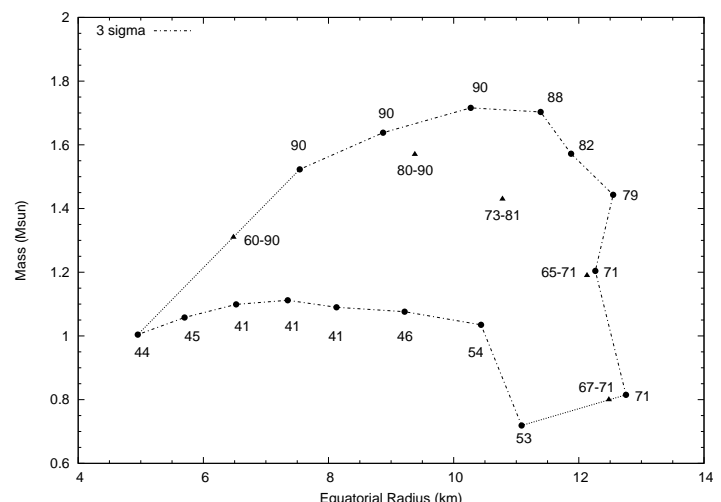

Figure 8. Inclination ranges for the joint fit to 1998B4 and 2002B3 data sets. Points labeled with a triangle correspond to the best-fit models shown in Table 3 For each one of these points, the $3 \sigma$ range of inclination angles (in degrees) for the given mass and radius is shown. The dot-dashed curve shows the same $3 \sigma$ limit on the allowed values of mass and radius shown in Figure 7 Selected points along the $3 \sigma$ contour are shown with circles. The inclination angle corresponding for each of these points is shown.

with bold curves in Figure 3 of Leahy et al. (2008) (labelled "oblate and time delays"). The $3 \sigma$ confidence region only allows very small stars with $R<7 \mathrm{~km}$ if only the 1998 epoch is used. The additional data from 2002B3 and allowance for scattered-light used in the present analysis allows stars with larger radii, extending to $13 \mathrm{~km}$ at the $3 \sigma$ confidence level. The largest mass stars allowed (at $3 \sigma$ ) have $M=1.7 M_{\odot}$. At $3 \sigma$, the data allow for a wide variety of modern equations of state.

\subsection{Inclination Constraints}

The best-fit models displayed in Table 3 have inclinations ranging from 70 - 90 degrees. However, for each best-fit set of values for mass and radius shown in Table 3 there is a range of inclination angles allowed by the data. The range of inclination angles allowed at the $3 \sigma$ level is found by keeping the mass and radius fixed at the values shown in the table, and allowing all other parameters to vary. In Figure 8, the locations on the mass-radius plane for the five models shown in Table 3 are marked with triangles. The allowed $3 \sigma$ range of inclination angles (in degrees) for each of these points is shown on Figure 8. For example, the model with $M=1.43 M_{\odot}$ and $R=10.8 \mathrm{~km}$ is allowed inclination angles in the range of $73^{\circ}<i<81^{\circ}$, although the lowest $\chi^{2}$ results for $i=75.5^{\circ}$.

On Figure 8 the same $3 \sigma$ contours on the mass-radius plane that were shown in Figure 7 are redisplayed. For selected points on the contours (shown with circles), the one value of inclination that is allowed is shown. For example, Figure 8 shows that the data allows at the 3 $\sigma$ confidence level a star with $M=1.44 M_{\odot}, R=12.5$ $\mathrm{km}$ and $i=79^{\circ}$. In general, the lowest mass stars require smaller inclination angles (near 40 degrees) while the highest mass stars require inclinations that are as high as is allowed.

There are a number of observations that suggest that the inclination angle may be smaller than 90 degrees. The strongest evidence is the absence of eclipses, which constrains $i<82^{\circ}$ assuming a Roche lobe filling companion (Chakrabarty \& Morgan 1998). Wang et al. (2001) observed V4580 Sgr, the optical counterpart of SAX J1808, during the 1998 outburst. They modelled the op- 


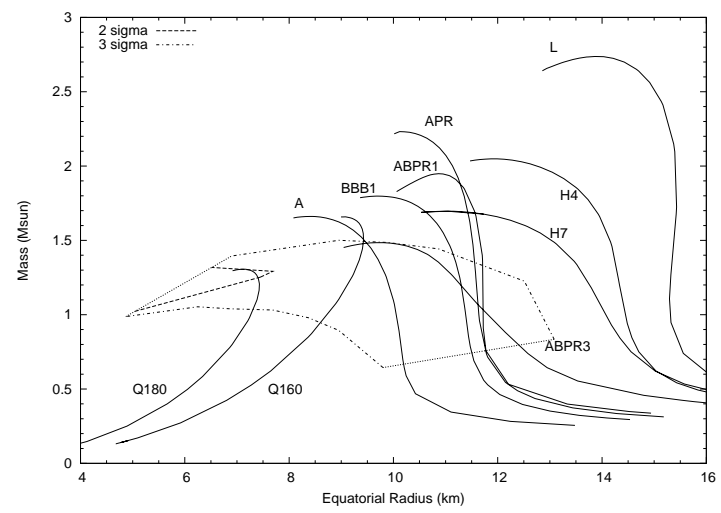

Figure 9. Mass and Radius confidence contours for joint fits to 1998B4 and 2002B3 data using the constraint $i \leq 70^{\circ}$. Massradius curves (solid curves) for stars spinning at a frequency of 401 $\mathrm{Hz}$ are displayed with solid curves. The radius corresponds to the equatorial radius of the star. EOS labels are the same as in Figure 7 Confidence contours for $2 \sigma$ (dashed curve) and $3 \sigma$ (dot-dashed curve) are shown.

tical and IR data using an X-ray-heated accretion disk and found limits on the inclination of $42^{\circ}<i<88^{\circ}$ if the distance is $d=2 \mathrm{kpc}$ and $20^{\circ}<i<65^{\circ}$ if $d=3$ kpc. Further modelling of the optical data observed during quiessence by Wang et al. (2009) suggests that $i<70^{\circ}$ using the distance of $d=3.5 \mathrm{kpc}$ derived by Gallowav \& Cumming (2006). Delove et al. (2008) modelled the optical data from quiessence using a model that includes emission from both the disk and the companion. Using a distance of $3.5 \mathrm{kpc}$, Deloye et al. (2008) constrain the inclination to $32^{\circ}<i<74^{\circ}$ if $10 \%$ uncertainty in the distance is assumed.

A feature interpreted as an iron line relativistically broadened through the orbital motion of the disk was detected in X-ray observations during the 2008 outburst by Cackett et al. (2009) and Papitto et al. (2009). Both groups modelled the emission feature and constrained the inclination angle. Cackett et al. (2009) found $i=55_{-4}^{+8}$ degrees, while Papitto et al. (2009) provide a less precise constraint of approximately $i>60^{\circ}$. A different type of constraint on the inclination arises from the analysis of the 2002 outburst data by Ibragimov \& Poutanen (2009). In their analysis, Ibragimov \& Poutanen (2009) assume that the X-ray emission is produced by two antipodal spots, one of which is sometimes hidden by the accretion disk, due to movement of the inner edge of the accretion disk. In this model, they find that the data can be explained by inclinations in the range $i<73^{\circ}$.

Although all of the constraints on inclination have some model dependence, there is a general agreement that inclinations smaller than about $70^{\circ}$ are suggested by the different data. With this in mind, we refit the data for 1998B4 and 2002B3 subject to the constraint $i \leq 70^{\circ}$. The best fit models for fixed values of $2 M / R$ are shown in Table 4. Comparing Table 4 with Table 3 it can be seen the the most significant change is that lower mass stars are allowed when the inclination angle is constrained to $i \leq 70^{\circ}$. The values of $\chi^{2}$ increase, but the magnitude of the increase is not significant.

Figure 9 shows the 2 and 3- $\sigma$ confidence contour on the mass-radius plane using the constraint $i \leq 70^{\circ}$ for joint fits to the 1998B 4 and 2002B3 data. The $3 \sigma$ confidence region shrinks when the constraint on inclination is added, however stars as massive as $1.5 M_{\odot}$ are still allowed.

\subsection{Location of the Hot Spot}

In all of the hot spot models for the 1998B4 and 2002B3 data sets, the colatitude of the spot $\theta$ is larger (further from the pole) in 1998 compared to the colatitude in 2002. This trend is seen in all models fitting the other data sets as well. Since a hot spot located close to the spin axis produces a less-modulated pulse shape, this is a natural outcome of the fact that the 1998 data is highly modulated $(\sim 10 \%)$ and the data for the later outbursts have smaller modulation $(\sim 5 \%)$.

In the best-fit models for the 1998B4 and 2002B3 data shown in Table 3 the shift in colatitude $\Delta \theta=\theta_{1}-\theta_{2}$ ranges from $5^{\circ}$ to $8^{\circ}$. When all models that fit the data within $3 \sigma$ confidence are considered, the colatitude in 1998 lies in the range $9^{\circ}<\theta_{1}<32^{\circ}$, the colatitude in 2002B3 lies in the range $4^{\circ}<\theta_{2}<17^{\circ}$. The $3 \sigma$ range of colatitude difference is in the range $6^{\circ}<\Delta \theta<15^{\circ}$. This wandering of the hot spot location is consistent with the model proposed by Lamb et al. (2009) where a hot spot close to the spin axis moves due to movement of the star's magnetic field.

\subsection{Joint Fits to $1998 B 4$ and 2002B4 Data}

The 2002B4 and 1998B4 data have similar pulse shapes (although a statistical test shows that they are different at high significance - chance probability $<10^{-20}$ ). Of all the data sets, these two epochs have the smallest errorbars which are roughly equal in magnitude. The most important difference is in the modulation of the pulse shapes.

The best-fit models for joint fits to the 1998B4 and 2002B4 data sets are shown in Table 5. In this table, the subscript " 2 " corresponds to values of in the 2002B4 epoch, while the subscript " 1 " corresponds to the 1998B4 epoch. These best-fit models should be compared with the best-fit models shown in Table 3 . The range of masses and radii found when the 2002B 4 data are fit is similar to those found when data from 2002B3 are used. The values of mass and radius shown in Table 5 are consistent with the $3 \sigma$ limits arising from the joint fit between the 1998B4 and 2002B3 data, with the exception of the model with a mass of $0.66 M_{\odot}$. The best-fit values of the 1998 angular location of the spot $\theta_{1}$ and the inclination angle $i$ differ in the best-fit models displayed in Tables 3 and 5. This is not surprising, since as we have shown earlier, given values of mass and radius, a wide range of angles $\theta$ and $i$ will give acceptable fits to the data.

The best-fit model's light curve for the 2002B4 data is shown in Figure 3. (The light curves for 1998B4 generated by the best-fit model are not distinguishable from the curves shown in Figure 1 and are not plotted.)

\subsection{Outburst Data}

The 2005 outburst was only about $70 \%$ as luminous as the 2002 outburst (Hartman et al. 2008). In addition, the RXTE satellite has lost sensitivity since 2002. As a result, the 2005 data is much noisier and has larger error bars than the 1998 and 2002 data as can be seen in Figures 4.6. One-spot models were used to simultaneously 
fit the 2005 data with the 1998 data. The best-fit model parameters are shown in Tables [6] and the best-fit light curves are shown in Figures 4,6.

The best-fit models that make use of the 2005 outburst data shown in Tables 6 - 8 are very similar to the best-fit models computed using the 2002B4 data and displayed in Table 5. The models with $2 M / R=0.4,0.5$ and 0.6 (and with $M>1.0 M_{\odot}$ ) are consistent with the $3 \sigma$ limits arising from the joint 1998B4-2002B3 fits.

The 2008 outburst was almost as bright as the 2005 outburst, however the degradation of the RXTE PCA over the intervening 3 years has the result that the pulse shapes from the 2008 outburst (Hartman et al. 2009), while similar to the 2005 pulse shapes, are even noisier than the 2005 pulse profiles. For this reason, we have not attempted to fit the 2008 pulse profiles.

\section{DISCUSSION}

In this paper we analyze multiple pulse shapes resulting from the different epochs of the 1998, 2002 and 2005 outbursts of SAX J1808. We jointly fit data from 1998 and several other epochs in order to find consistent models for the neutron star's mass, radius and inclination.

In each joint fit to the data from two different epochs, the mass, equatorial radius and the inclination angle are kept fixed between epochs, while the spot location, anisotropy and blackbody-to-compton ratios are allowed to change from epoch to epoch. We use a spectral model, motivated by Gierliński et al. (2002) and Poutanen \& Gierliński (2003), that includes blackbody radiation and Comptonized radiation in the low-energy (3-4 keV) band and only Comptonized radiation in the high-energy (9-20 keV) band. We find that an extra scattered-light component is required to fit all of the pulse shapes.

The inclusion of the scattered radiation model (Section 3.2), together with a hot spot that wanders a little in its co-latitude, allows a consistent fit for all different epochs with a consistent mass. Increased radius or increased spot co-latitude increases the second harmonic content in the pulse shape; scattered radiation reduces it; and increased light bending (smaller star at a given mass) can increase or decrease second harmonic content depending on viewing geometry. Without including all these physical effects, the fits to some epochs require a small star whereas other epochs require a larger star. As we have shown, by including all effects, we can consistently fit different epochs with a consistent mass.

The joint fit of data from the 1998B4 and 2002B3 epochs using the constraint $i<90^{\circ}$ leads to the $3 \sigma$ confidence constraint on the neutron star mass $0.81 M_{\odot}<$ $M<1.72 M_{\odot}$ and equatorial radius $5.0 \mathrm{~km}<R<$ $12.7 \mathrm{~km}$, as summarized in Figure 7 . Inclinations as low as $41^{\circ}$ are allowed. If the inclination is further constrained to $i<70^{\circ}$, as suggested by many separate observations, the $3 \sigma$ limits are $0.83 M_{\odot}<M<1.50 M_{\odot}$ and $7 \mathrm{~km}<R<13 \mathrm{~km}$, as summarized in Figure 9. Inclinations as low as $35^{\circ}$ are allowed in this case.

The joint fits between 1998B4 and the other epochs (2002B4, 2005B1, 2005B2 and 2005B4) yield independent best-fit models summarized in Tables 5,8. The bestfit models from these epochs with $M>1.0 M_{\odot}$ have mass and radius values that lie within the $3 \sigma$ confidence region for the 1998B4-2002B3 joint fits. This indicates that the various joint fits between the different epochs give consistent results.

The $3 \sigma$ confidence region for the 1998B4-2002B3 joint fits allows a much wider range of masses and radii for the neutron star than a similar fit that only made use of data from the 1998 outburst (Leahy et al. 2008). In the previous analysis by Leahy et al. (2008) a similar type of analysis using 2 narrow energy bands constrained the neutron star mass and radius to very small values with $R<7 \mathrm{~km}$. The inclusion of a scattered-light component and use of joint fits with data from other epochs (2002 and 2005 outbursts) results in stars with larger masses and radii than are given by fits to the 1998 data alone without the scattered-light component.

In their analysis of the 2002 data, Ibragimov \& Poutanen (2009) do not attempt to find a best-fit mass and radius for SAX J1808. For some of their models they make use of a fixed mass of 1.4 $M_{\odot}$ and a radius of either 10 or $12 \mathrm{~km}$. However, the results of their modelling (such as the magnetic moment, visibility of an antipodal spot) do not strongly depend on the assumed values of mass and radius. A mass of $1.4 M_{\odot}$ and a radius in the $10-12 \mathrm{~km}$ range is consistent with our final results.

Observations of SAX J1808 in quiescence by Heinke et al. (2007, 2009) have set limits on the luminosity. The low luminosity during quiescence implies that rapid neutrino cooling takes place in the inner core, such as the direct URCA process. Yakovlev \& Pethick (2004) show that direct URCA can take place if the core density is larger than approximately $10^{15} \mathrm{~g} / \mathrm{cm}^{3}$. For a standard hadronic EOS such as APR (Akmal et al. 1998), stars with masses larger than about $1.5 M_{\odot}$ (and spinning at $400 \mathrm{~Hz}$ ) have central densities larger than this critical density. Softer EOS will have lower mass stars for the same central density. This suggests that many of the models allowed by the $3 \sigma$ confidence region shown in Figure 7 could have cores dense enough to allow cooling through the direct URCA process.

A pulse-shape analysis of the accreting ms-period Xray pulsar XTE 1814-338 (Leahy et al. 2009) using the similar methods as used in this paper implied that the mass and radius of XTE 1814-338 must be quite large. Although the $3 \sigma$ regions for XTE 1814-338 and SAX J1808 do not overlap, the results are not necessarily inconsistent. It is only necessary for an EOS mass-radius curve to pass through the regions for both stars. For example, an EOS mass-radius curve that is slightly to the right of APR in Figure 7 would still be allowed by the SAX J1808 data, and would be stiff enough to be allowed by the XTE 1814 data, and also allowed by the constraints on the slow X-ray pulsar Hercules X-1 (Leahy 2004).

This research was supported by grants from NSERC. We thank Jake Hartman for supplying us with the pulse profiles used in this analysis, and for helpful comments on the paper.

\section{REFERENCES}

Akmal, A., Pandharipande, V. R., \& Ravenhall, D. G. 1998, Phys. Rev. C, 58, 1804

Alford, M., Braby, M., Paris, M., \& Reddy, S. 2005, ApJ 629, 969 
Arnett, W. D., \& Bowers, R. L. 1977, ApJS, 33, 415

Baldo, M., Bombaci, I., \& Burgio, G. F., 1997, A\&A, 328, 274

Cackett, E. M., Altamirano, D., Patruno, A., Miller, J. M., Reynolds, M., Linares, M., \& Wijnands, R. 2009, ApJ, 694, L21

Cadeau, C., Leahy, D. A., \& Morsink, S. M. 2005, ApJ, 618, 451

Cadeau, C., Morsink, S. M., Leahy, D. A., \& Campbell, S. S. 2007, ApJ, 654, 458

Chakrabarty, D. \& Morgan, E. H. 1998, Nature, 394, 346

Deloye, C. J., Heinke, C. O., Taam, R. E., \& Jonker, P. G. 2008, MNRAS, 391, 1619

Galloway, D. K., \& Cumming, A. 2006, ApJ, 652, 559

Gierliński, M., Done, C., \& Barret, D. 2002, MNRAS, 331, 141

Glendenning, G. K. 2000, Compact Stars, (Springer-Verlag, New York)

Hartman, J. M., et al. 2008, ApJ, 675, 1468

Hartman, J. M., et al. 2009, ApJ, 702, 1673

Heinke, C. O., Jonker, P. G., Wijnands, R., \& Taam, R. E. 2007, ApJ, 660, 1424

Heinke, C. O., Jonker, P. G., Wijnands, R., Deloye, C. J., \& Taam, R. E. 2009, ApJ, 691, 1035

Ibragimov, A., \& Poutanen, J. 2009, MNRAS, 400, 492

Lackey, B. D., Nayyar, M., \& Owen, B. J. 2006, Phys. Rev. D, 73, 024021

Lamb, F. K., Boutloukos, S., Van Wassenhove, S., Chamberlain, R. T., Lo, K. H., Clare, A., Yu, W., \& Miller, M. C. 2009, ApJ, 706,417
Leahy, D. A. 2004, ApJ, 613, 517

Leahy, D. A., Morsink, S. M., \& Cadeau, C. 2008, ApJ, 672, 1119

Leahy, D. A., Morsink, S. M., Chung, Y.-Y., \& Chou, Y. 2009, ApJ, 691, 1235

Morsink, S. M., Leahy, D. A., Cadeau, C. \& Braga, J. 2007, ApJ, 663,1244

Pandharipande, V. R. 1971, Nucl. Phys. A, 174, 641

Pandharipande, V. R., Pines D., \& Smith, R. A. 1976, ApJ, 208, 550

Papitto, A., Menna, M. T., Burderi, L., Di Salvo, T., D’Antona, F., \& Robba, N. R. 2005, ApJ, 621, L113

Papitto, A., Di Salvo, T., D'Aì, A., Iaria, R., Burderi, L., Riggio, A., Menna, M. T., \& Robba, N. R. 2009, A\&A, 493, L39

Poutanen, J. \& Gierliński, M. 2003, MNRAS, 343, 1301

Poutanen, J. 2006, Advances in Space Research, 38, 2697

Sazonov, S. Y. \& Sunyaev, R. A. 2001, A\&A, 373, 241

Wang, Z., et al. 2001, ApJ, 563, L61

Wang, Z., Bassa, C., Cumming, A., \& Kaspi, V. M. 2009, ApJ, 694,1115

Wijnands, R., \& van der Klis, M. 1998, Nature, 394, 344

Wijnands, R. 2006, Trends in Pulsar Research, 53

Yakovlev, D. G., \& Pethick, C. J. 2004, ARA\&A, 42, 169 
Table 1

Effect of Spot Size and Shape for stellar models with $2 M / R=0.50$ fit with data from $2002 \mathrm{~B} 3$.

\begin{tabular}{|c|c|c|c|c|c|c|c|}
\hline Model & $\begin{array}{c}M \\
M_{\odot}\end{array}$ & $\chi^{2} /$ dof & $\begin{array}{c}\theta \\
\text { deg. }\end{array}$ & $\begin{array}{c}i \\
\text { deg. }\end{array}$ & $a$ & $\theta_{2}$ & $d \phi$ \\
\hline infinitesimal spot & 0.304 & $69.7 / 56$ & 27.0 & 36.0 & 0.50 & & \\
\hline circle radius $0.1 R$ & 0.305 & $69.7 / 56$ & 27.0 & 36.2 & 0.51 & & \\
\hline circle radius $0.2 R$ & 0.307 & $69.7 / 56$ & 27.2 & 36.6 & 0.51 & & \\
\hline azimuthal line $0.4 R$ & 0.310 & $69.2 / 56$ & 26.2 & 35.8 & 0.51 & & \\
\hline two spots (equal amp.) & 0.354 & $62.1 / 54$ & 12.0 & 26.0 & 0.54 & 54.4 & 60.1 \\
\hline
\end{tabular}

Table 2

Best Fit Parameters for Joint Fits using Data from 1998B4 and 2002B3 with and without the scattered light component for $2 M / R=0.5$.

\begin{tabular}{|c|c|c|c|c|c|c|c|c|c|c|c|}
\hline & $\begin{array}{c}M \\
M_{\odot}\end{array}$ & $\begin{array}{c}R \\
\mathrm{~km}\end{array}$ & $\begin{array}{c}\theta_{1} \\
\text { deg. }\end{array}$ & $\begin{array}{c}\theta_{2} \\
\text { deg. }\end{array}$ & $\begin{array}{c}i \\
\text { deg. }\end{array}$ & $a_{1}$ & $a_{2}$ & $I_{s 1}$ & $I_{s 2}$ & $\begin{array}{c}r \\
\mathrm{~km}\end{array}$ & $\chi^{2} /$ dof \\
\hline No Scatter & 1.02 & 6.0 & 22.6 & 10.6 & 49.5 & 0.57 & 0.49 & 0 & 0 & & $200 / 113$ \\
\hline Model 1 & 1.44 & 8.5 & 12.0 & 5.6 & 80.0 & 0.80 & 0.68 & 0.002 & 0.008 & 116 & $120 / 110$ \\
\hline Model 2 & 1.57 & 9.4 & 10.5 & 4.8 & 88.9 & 0.92 & 0.77 & 0.003 & 0.007 & & $120 / 110$ \\
\hline
\end{tabular}

Note. - Subscript "1" corresponds to 1998B4 values and "2" to 2002 B3.

Table 3

Best Fit Parameters for Joint Fits using Data from 1998B4 and 2002B3.

\begin{tabular}{|c|c|c|c|c|c|c|c|c|}
\hline $2 M / R$ & $\begin{array}{c}M \\
M_{\odot} \\
\end{array}$ & $\begin{array}{c}R \\
\mathrm{~km}\end{array}$ & $\begin{array}{c}\theta_{1} \\
\text { deg. }\end{array}$ & $\begin{array}{c}\theta_{2} \\
\mathrm{deg} .\end{array}$ & $\begin{array}{c}i \\
\text { deg. }\end{array}$ & $a_{1}$ & $a_{2}$ & $\chi^{2} /$ dof \\
\hline 0.60 & 1.31 & 6.5 & 14.3 & 6.2 & 89.6 & 0.77 & 0.62 & $116 / 110$ \\
\hline 0.50 & 1.57 & 9.4 & 10.5 & 4.8 & 88.9 & 0.92 & 0.77 & $120 / 110$ \\
\hline 0.40 & 1.43 & 10.8 & 10.4 & 4.9 & 75.5 & 0.85 & 0.73 & $123 / 110$ \\
\hline 0.30 & 1.19 & 12.1 & 10.0 & 4.7 & 70.8 & 0.89 & 0.75 & $124 / 110$ \\
\hline 0.20 & 0.80 & 12.5 & 10.2 & 5.0 & 70.3 & 1.0 & 0.88 & $123 / 110$ \\
\hline
\end{tabular}

Note. - Subscript "1" corresponds to 1998B4 values and "2" to 2002 B3.

Table 4

Best Fit Parameters for Joint Fits using Data from 1998B4 and 2002B3 with $i \leq 70^{\circ}$.

\begin{tabular}{|c|c|c|c|c|c|c|c|c|}
\hline $2 M / R$ & $\begin{array}{c}M \\
M_{\odot}\end{array}$ & $\begin{array}{c}R \\
\mathrm{~km}\end{array}$ & $\begin{array}{c}\theta_{1} \\
\text { deg. }\end{array}$ & $\begin{array}{c}\theta_{2} \\
\text { deg. }\end{array}$ & $\begin{array}{c}i \\
\text { deg. }\end{array}$ & $a_{1}$ & $a_{2}$ & $\chi^{2} /$ dof \\
\hline 0.60 & 1.21 & 6.0 & 18.4 & 8.3 & 70.0 & 0.64 & 0.52 & $118 / 110$ \\
\hline 0.50 & 1.27 & 7.6 & 16.0 & 7.6 & 63.3 & 0.65 & 0.56 & $122 / 110$ \\
\hline 0.40 & 1.30 & 9.7 & 12.7 & 6.1 & 64.8 & 0.72 & 0.62 & $124 / 110$ \\
\hline 0.30 & 1.09 & 11.0 & 11.9 & 5.8 & 61.9 & 0.75 & 0.65 & $125 / 110$ \\
\hline 0.20 & 0.80 & 12.5 & 10.2 & 5.0 & 70.0 & 0.99 & 0.87 & $123 / 110$ \\
\hline
\end{tabular}

Note. - Subscript "1" corresponds to 1998B4 values and "2" to 2002 B3. 
Table 5

Best Fit Parameters for Joint Fits using Data from 1998B4 and 2002B4.

\begin{tabular}{ccccccccc}
\hline \hline $2 M / R$ & $\begin{array}{c}M \\
M_{\odot}\end{array}$ & $\begin{array}{c}R \\
\mathrm{~km}\end{array}$ & $\begin{array}{c}\theta_{1} \\
\mathrm{deg} .\end{array}$ & $\begin{array}{c}\theta_{2} \\
\mathrm{deg} .\end{array}$ & $\begin{array}{c}i \\
\mathrm{deg} .\end{array}$ & $a_{1}$ & $a_{2}$ \\
& 1.27 & 6.3 & 22.3 & 18.6 & 53.1 & 0.57 & 0.65 \\
0.60 & 1.27 & 7.5 & 21.0 & 17.5 & 46.9 & 0.57 & 0.64 \\
0.50 & 1.19 & 8.9 & 20.2 & 16.6 & 41.9 & 0.57 & 0.64 \\
0.30 & 0.96 & 9.7 & 19.5 & 16.3 & 39.4 & 0.57 & 0.64 \\
0.20 & 0.66 & 10.1 & 19.2 & 16.4 & 38.3 & 0.58 & 0.65 & $111 / 110$ \\
& & & & & & & & \\
\hline
\end{tabular}

Note. - Subscript "1" corresponds to 1998B4 values and "2" to 2002B4.

Table 6

Best Fit Parameters for Joint Fits using Data from 1998B4 and 2005B1.

\begin{tabular}{|c|c|c|c|c|c|c|c|c|}
\hline $2 M / R$ & $\begin{array}{c}M \\
M_{\odot}\end{array}$ & $\begin{array}{c}R \\
\mathrm{~km}\end{array}$ & $\begin{array}{c}\theta_{1} \\
\text { deg. }\end{array}$ & $\begin{array}{c}\theta_{2} \\
\text { deg. }\end{array}$ & $\begin{array}{c}i \\
\text { deg. }\end{array}$ & $a_{1}$ & $a_{2}$ & $\chi^{2} /$ dof \\
\hline 0.60 & 1.36 & 6.7 & 19.6 & 19.2 & 57.2 & 0.58 & 0.66 & $111 / 110$ \\
\hline 0.50 & 1.33 & 7.9 & 18.8 & 18.6 & 49.9 & 0.58 & 0.65 & $114 / 110$ \\
\hline 0.40 & 1.20 & 9.0 & 18.3 & 18.1 & 44.8 & 0.58 & 0.65 & $116 / 110$ \\
\hline 0.30 & 0.98 & 9.9 & 18.0 & 17.9 & 41.6 & 0.58 & 0.65 & $117 / 110$ \\
\hline 0.20 & 0.67 & 10.3 & 17.9 & 17.9 & 40.1 & 0.58 & 0.66 & $119 / 110$ \\
\hline
\end{tabular}

Note. - Subscript "1" corresponds to 1998B4 values and "2" to 2005B1.

Table 7

Best Fit Parameters for Joint Fits using Data from 1998B4 and 2005B2.

\begin{tabular}{cccccccc}
\hline \hline $2 M / R$ & $\begin{array}{c}M \\
M_{\odot}\end{array}$ & $\begin{array}{c}R \\
\mathrm{~km}\end{array}$ & $\begin{array}{c}\theta_{1} \\
\mathrm{deg}\end{array}$ & $\begin{array}{c}\theta_{2} \\
\mathrm{deg} .\end{array}$ & $\begin{array}{c}i \\
\mathrm{deg} .\end{array}$ & $a_{1}$ & $a_{2}$ \\
& 1.20 & 5.9 & 23.1 & 13.9 & 53.2 & 0.57 & 0.64 \\
0.60 & 1.20 & 7.1 & 21.9 & 13.1 & 46.6 & 0.57 & 0.63 \\
0.50 & 1.09 & 8.1 & 21.1 & 12.5 & 42.1 & 0.57 & 0.62 \\
0.40 & 0.90 & 9.1 & 20.5 & 12.3 & 39.0 & 0.57 & 0.62 \\
0.30 & 0.64 & 9.7 & 20.1 & 12.0 & 37.5 & 0.58 & 0.63 \\
0.20 & & & & & & & $111 / 110$ \\
& & & & & & & \\
\hline
\end{tabular}

Note. - Subscript "1" corresponds to 1998 B4 values and "2" to 2005B2.

Table 8

Best Fit Parameters for Joint Fits using Data from 1998B4 and 2005B4.

\begin{tabular}{ccccccccc}
\hline \hline $2 M / R$ & $M$ & $\begin{array}{c}R \\
\mathrm{~km}\end{array}$ & $\begin{array}{c}\theta_{1} \\
\mathrm{deg} .\end{array}$ & $\begin{array}{c}\theta_{2} \\
\mathrm{deg} .\end{array}$ & $\begin{array}{c}i \\
\mathrm{deg} .\end{array}$ & $a_{1}$ & $a_{2}$ & $\chi^{2} / \mathrm{dof}$ \\
\hline 0.60 & 1.19 & 5.9 & 23.0 & 11.3 & 54.4 & 0.57 & 0.64 \\
0.50 & 1.18 & 7.0 & 21.9 & 10.7 & 47.3 & 0.57 & 0.63 & $109 / 110$ \\
0.40 & 1.07 & 8.0 & 21.1 & 10.3 & 42.5 & 0.57 & 0.63 & $111 / 110$ \\
0.30 & 0.89 & 8.9 & 20.3 & 9.9 & 40.0 & 0.58 & 0.63 & $114 / 110$ \\
0.20 & 0.63 & 9.6 & 19.9 & 9.7 & 38.3 & 0.58 & 0.64 & $115 / 110$ \\
& & & & & & & & \\
\hline
\end{tabular}

Note. - Subscript "1" corresponds to 1998B4 values and "2" to 2005B4. 